\title{
Hybrid UV Curing System Using Methacrylates Having a Chalcone Moiety
}

\author{
Haruyuki Okamura, Yuta Ueda and Masamitsu Shirai \\ Department of Applied Chemistry, Graduate School of Engineering, \\ Osaka Prefecture University, 1-1 Gakuen-cho, Nakaku, Sakai, Osaka 599-8531, Japan \\ okamura@chem.osakafu-u.ac.jp
}

Keywords: hybrid, UV curing, chalcone, methacrylate

\section{Introduction}

Hybrid UV curing system, which uses two distinguished UV curing reactions independently or simultaneously, has attracted much attention due to high sensitivity, decreased oxygen inhibition, and spatial control of hardness and flexibility [1-14]. Especially, radical/cation hybrid system using a photoradical initiator and a photoacid generator was extensively studied due to easy access of monomers and initiators [1-9]. For example, blends of acrylate monomers, which polymerize by a radical mechanism and epoxy functionalized monomers, which polymerize by a cationic mechanism, are irradiated to produce networks [1-9]. Monomer structures combined with photoradical initiators, photoacid generators, and sensitizers have been studied to control the cationic polymerization and radical polymerization. Comprehensive understanding of the radical/cation hybrid system is difficult due to the complexity of the system.

We employed photodimerization reaction instead of cationic polymerization in hybrid UV curing system. Photodimerization of anthracene, cinnmate ester, chalcone, and coumarin proceed by irradiation of UV light $[15,16]$. Photodimerization conditions are tunable by selecting the dimerization moieties and/or irradiation conditions.

In this work, we have designed and synthesized a novel dimethacrylate monomer having a chalcone moiety. The monomer was applied to hybrid UV curing system using photoradical polymerization of methacrylates and photodimerization of chalcone as shown in Fig. 1. The effect of photoradical initiators and irradiation wavelength was investigated to control photodimerization and photoradical polymerization. Reaction mechanism of the system was discussed. Application of the system to photocuring system with reduced oxygen inhibition was also carried out.

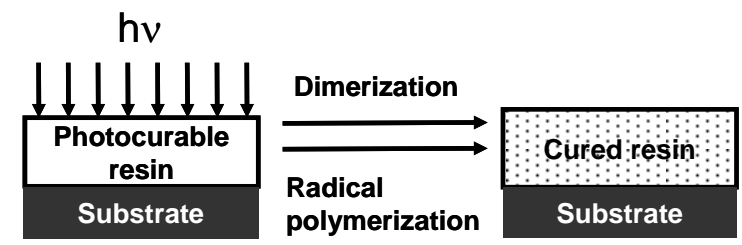

Fig. 1. Schematic illustration of hybrid UV curing system in this work.

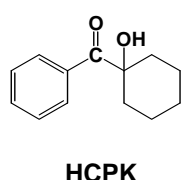

HCPK

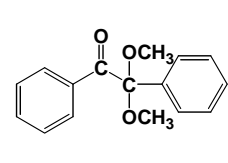

DMPA

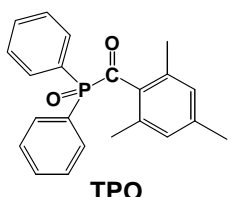

TPO

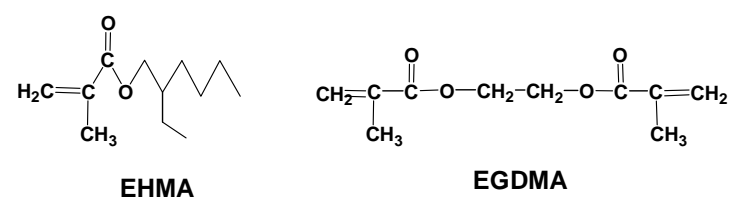

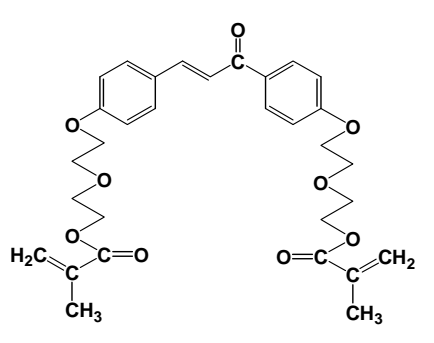

CDMA

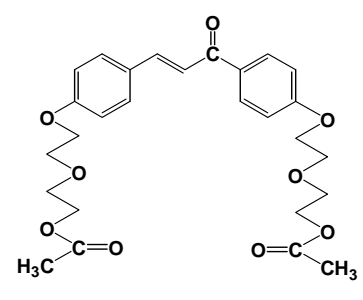

CDAC
Fig. 2. Structures of chemicals used. 


\section{Experimental}

\subsection{Materials}

Structures of chemicals used were shown in Fig.

2. Photoradical initiators 1-hydroxy-1-cyclohexyl phenyl ketone (HCPK), 2,2-dimethoxy-2-phenylacetophenone (DMPA), and 2,4,6-trimethylbenzoyldiphenylphosphine oxide (TPO) were used as received. As diluent monomers, 2-ethylhexyl methacrylate (EHMA) and ethyleneglycol dimethacrylate (EGDMA) were purchased and used without further purification.

A dimethacryalate having a chalcone moiety (CDMA) was prepared as shown in Scheme 1.

A chalcone moiety, 1,3-bis(4-hydroxyphenyl)propenone (BHPP) [17] was synthesized by cationic aldol condensation.
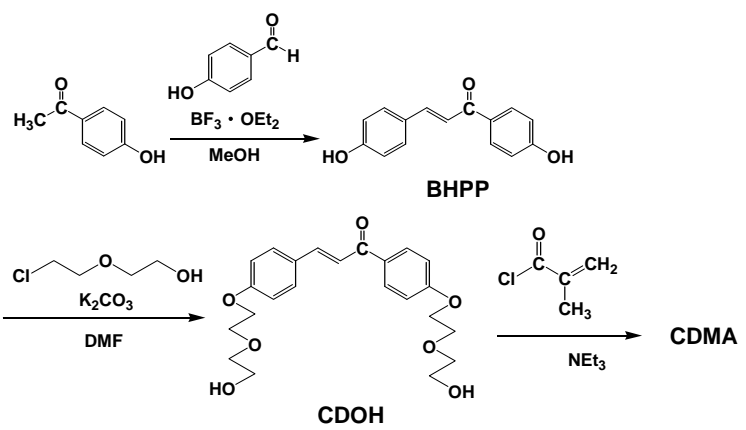

Scheme 1. Synthesis of CDMA.

BHPP (10.54 g, 43.9 mmol), 2-(2-chloroethoxy)ethanol (11.16 g, $89.6 \mathrm{mmol})$, and sodium carbonate $(6.28 \mathrm{~g}, 45.5 \mathrm{mmol})$ were mixed in $N, N$-dimethylformamide (DMF) $(80 \mathrm{~mL})$. The mixture was heated at $100{ }^{\circ} \mathrm{C}$ for $65 \mathrm{~h}$ with stirring. The reaction mixture was pored into ice-cold water and extracted with chloroform. The chloroform solution was washed with deionized water twice, and dried. The product 1,3-bis $\{4-[2-(2-$ hydroxyethoxy)ethoxy]phenyl\}propenone $(\mathrm{CDOH})$ was obtained after column chromatography (eluent; AcOEt/THF $=8 / 2$ ) as white solid; yield $5.25 \mathrm{~g}$ (29\%). mp: $75 \sim 78 \quad{ }^{\circ} \mathrm{C} . \quad{ }^{1} \mathrm{H} \quad \mathrm{NMR} \quad\left(\mathrm{CDCl}_{3}\right)$ : 8 8.0-6.9 (8H, m, aromatic), 7.8-7.4 $(2 \mathrm{H}, \mathrm{d}$, $-\mathrm{CH}=\mathrm{CH}-), 4.2-3.6\left(16 \mathrm{H}, \mathrm{m}, \mathrm{CH}_{2}\right) . \quad \mathrm{MS}(\mathrm{EI}), \mathrm{m} / \mathrm{z}$ 416( $\left.\mathrm{M}^{+}, 38.2\right), 45\left(\mathrm{CH}_{3}-\mathrm{CH}=\mathrm{OH}^{+}, 100\right)$.

$\mathrm{CDOH}(10.54 \mathrm{~g}, 43.9 \mathrm{mmol})$ was dissolved in chloroform (60 mL). Triethylamine (10.54 g, 43.9 mmol) was added in the mixture. The mixture was cooled using ice-cold bath. After cooling, methacryloyl chloride (10.54 g, $43.9 \mathrm{mmol})$ was added dropwise in the mixture. The mixture was stirred for $6 \mathrm{~h}$ at ambient temperature. The reaction mixture was pored into ice-cold water and extracted with chloroform. The chloroform layer was washed with saturated sodium bicarbonate solution twice and deionized water twice, and dried. The product 1,3-bis\{4-[2-(2-methacryloxyethoxy)ethoxy]phenyl \}propenone (CDMA) was obtained after column chromatography (eluent; AcOEt $/ \mathrm{CHCl}_{3}=8 / 2$ ) as yellow gammy solid; yield $3.70 \mathrm{~g}(48 \%) .{ }^{1} \mathrm{H}$ NMR $\left(\mathrm{CDCl}_{3}\right): \delta 8.0-6.9(8 \mathrm{H}$, $\mathrm{m}$, aromatic), 7.8-7.4 (2H, d, $-\mathrm{CH}=\mathrm{CH}-), 6.1(2 \mathrm{H}$, $\mathrm{s}$, methacryl $\left.\mathrm{CH}_{2}\right), 5.6\left(2 \mathrm{H}\right.$, s, methacryl $\left.\mathrm{CH}_{2}\right)$, 4.4-3.8 (16H, m, $\left.\mathrm{CH}_{2}\right), 1.9$ (6H, s, methacryl $\left.\mathrm{CH}_{3}\right)$. MS (EI), m/z $552\left(\mathrm{M}^{+}, 17.3\right), 69\left(\mathrm{CH}_{2}=\mathrm{C}\left(\mathrm{CH}_{3}\right) \mathrm{CO}^{+}\right.$, 100). Anal. Calcd for $\mathrm{C}_{31} \mathrm{H}_{36} \mathrm{O}_{9}: \mathrm{C}, 67.38 ; \mathrm{H}$, 6.57. Found: C, 66.53; H, 6.53.

The model compound 1,3-bis\{4-[2-(2-acetoxyethoxy)ethoxy]phenyl\}propenone (CDAc) was synthesized using $\mathrm{CDOH}$ and acetic anhydride in the same manner of the synthesis of CDMA. CDAc was purified by column chromatography (eluent; $\mathrm{CHCl}_{3}$ ). Yellow liquid; yield $36 \%$. ${ }^{1} \mathrm{H}$ NMR $\left(\mathrm{CDCl}_{3}\right): \delta$ 8.0-6.9 (8H, $\mathrm{m}$, aromatic), 7.8-7.4 (2H, d, - $\mathrm{CH}=\mathrm{CH}-)$, 4.4-3.8 $\left(16 \mathrm{H}, \mathrm{m}, \mathrm{CH}_{2}\right)$, $2.6\left(6 \mathrm{H}\right.$, s, acetyl $\left.\mathrm{CH}_{3}\right)$. MS (EI), m/z $500\left(\mathrm{M}^{+}\right.$, 20.4), $43\left(\mathrm{CH}_{3} \mathrm{CO}^{+}, 100\right)$. Anal. Calcd for $\mathrm{C}_{27} \mathrm{H}_{32} \mathrm{O}_{9}$ : C, 64.79; H, 6.44. Found: C, 63.94; H, 6.48 .

\subsection{Method}

A mixture of CDMA, EHMA or EDGMA, and a photoradical initiator was sandwiched by $\mathrm{CaF}_{2}$ plates. Irradiation was performed using a Xenon lamp (Asahi Spectra, MAX-301, $300 \mathrm{~W}$ ) through bandpath filters at 254 or $365 \mathrm{~nm}$ or a sharpcat filter at $370 \mathrm{~nm}$. Irradiation at $405 \mathrm{~nm}$ was performed using a LED laser (Ball Semiconductor, BP300, $300 \mathrm{~mW}$ ). Intensity at the light was measured with an Orc Light Measure UV-M02. Conversion of the methacryl unit and the chalcone unit in the blended film was determined by FT-IR measurements using the peak at $1638 \mathrm{~cm}^{-1}$ ascribed to the methacryl unit and $1602 \mathrm{~cm}^{-1}$ ascribed to the chalcone unit, respectively.

\subsection{Measurements}

${ }^{1} \mathrm{H}$ NMR spectra were measured by a JEOL ECX400 spectrometer. FT-IR measurements were carried out using a JASCO IR-410. UV-vis spectra were taken on a Shimadzu UV-2400 PC.

\section{Results and discussion}

Methacrylates are widely used as UV curing materials due to high reactivity. However, oxygen inhibition is a serious problem. On the other hand, chalcone takes place photodimerization 
even in air. Photodimerization behavior of chalcone moieties was investigated and applied to photosensitive polymers [18-21]. We have deigned and synthesized a dimethacrylate containing a chalcone moiety (CDMA) (Fig. 2). The methacryl units and the chalcone unit are connected with oxyethylene units which enhanced reactivity and the compatibility to additives.

The effect of photoradical initiators or irradiation wavelength on the conversion of the methacryl unit and the chalcone unit was investigated using UV-vis and FT-IR spectroscopy.

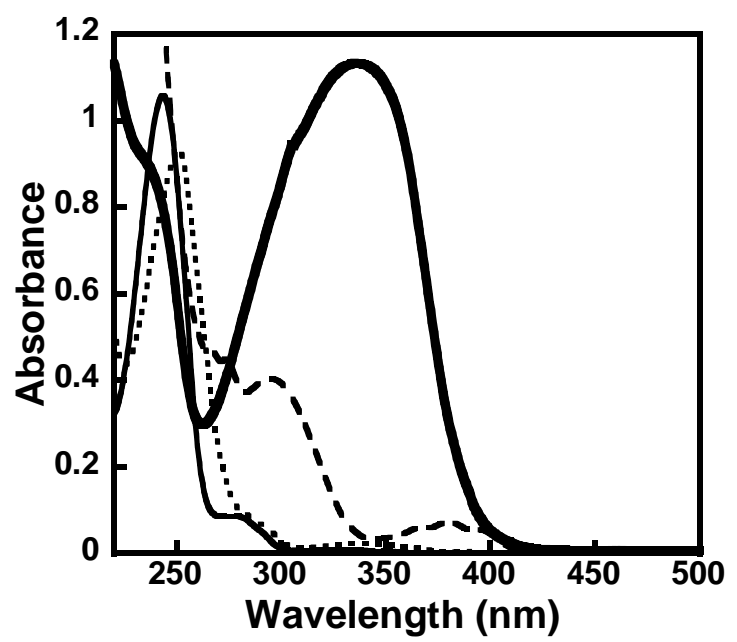

Fig. 3. UV-vis spectrum of $0.1 \mu \mathrm{m}$-thick CDMA/EHMA (1:1, wt/wt) blended film (bold line). UV-vis spectra of TPO (broken line), DMPA (dotted line) and HCPK (solid line) in acetonitrile $\left(1.0 \times 10^{-4} \mathrm{M}\right)$ were also shown.

Figure 3 shows UV-vis spectrum of 0.1 $\mu$ m-thick CDMA/EHMA (1/1, wt/wt) blended film. Strong absorption at around $350 \mathrm{~nm}$ ascribed to a chalcone moiety was observed. On irradiation at $365 \mathrm{~nm}$, complete bleaching of the absorption occurred. Absorption of the same concentration of TPO, DMPA, and HCPK at $254 \mathrm{~nm}$ was nearly identical to each other. On the other hand, absorption of the same concentration of the photoradical initiators at $365 \mathrm{~nm}$ was in the order, TPO $>$ DMPA $>$ HCPK.

Figure 4 shows the conversion of the methacryl unit and the chalcone unit in CDMA/EHMA/HCPK (1/1/0.06, wt/wt) blended film on irradiation at $365 \mathrm{~nm}$. The reaction rate of the chalcone unit was larger than that of methacryl unit at the initial stage. When the film was irradiated at $254 \mathrm{~nm}$, the reaction rate of the chalcone unit was smaller than that of the methacryl unit. When the film was irradiated at
370 400 $\mathrm{nm}$, the reaction rate of the chalcone moiety and the methacryl unit was similar to each other. Thus, we have found that the conversion of the methacryl unit and the chalcone unit was controllable by the irradiation wavelength.

When using TPO and DMPA as photoradical initiators, the reaction rate of the chalcone unit was smaller than that of methacryl unit on irradiation at $254 \mathrm{~nm}, 365 \mathrm{~nm}$, and 370-400 nm in all cases. Using $405 \mathrm{~nm}$ light, the methacryl unit only reacted.

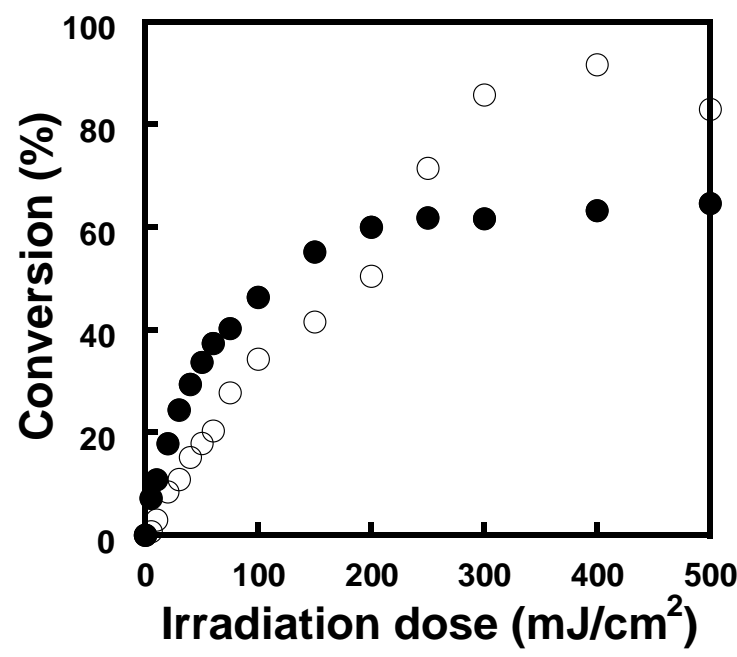

Fig. 4. Conversion of methacryl $(\mathrm{O})$ and chalcone $(\bullet)$ units of CDMA/EHMA/HCPK (1/1/0.06, wt/wt) blended film on irradiation at $365 \mathrm{~nm}$.

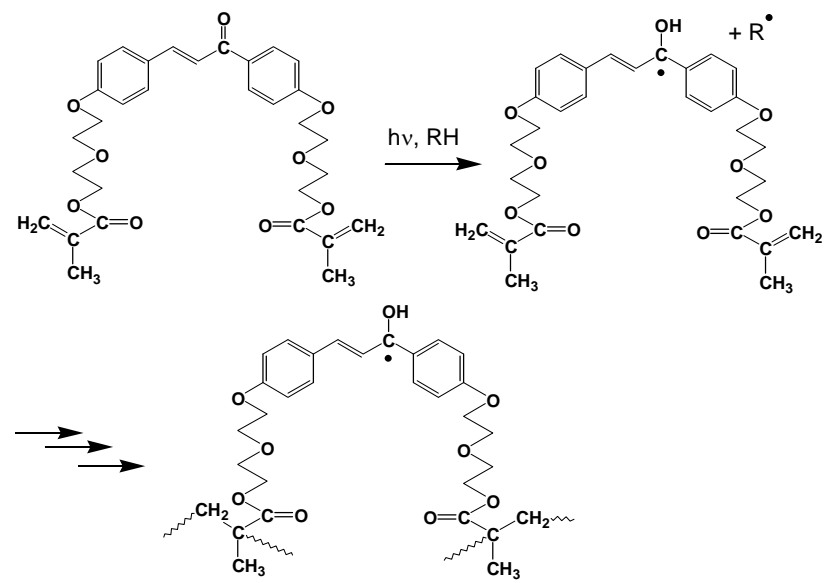

Scheme 2. Plausible reaction mechanism of radical polymerization of methacryl units initiated by CDMA on irradiation.

Photoradical polymerization of CDMA/EHMA (1/1, wt/wt) blended film proceeded on irradiation without photoradical initiators (Scheme 2). The 
reaction rate of the methacrylate became larger on addition of triethanol amine. CDAc also initiated the photopolymerization of EHMA. CDMA and CDAc may act as a type II photoradical initiator as shown in Scheme 2.

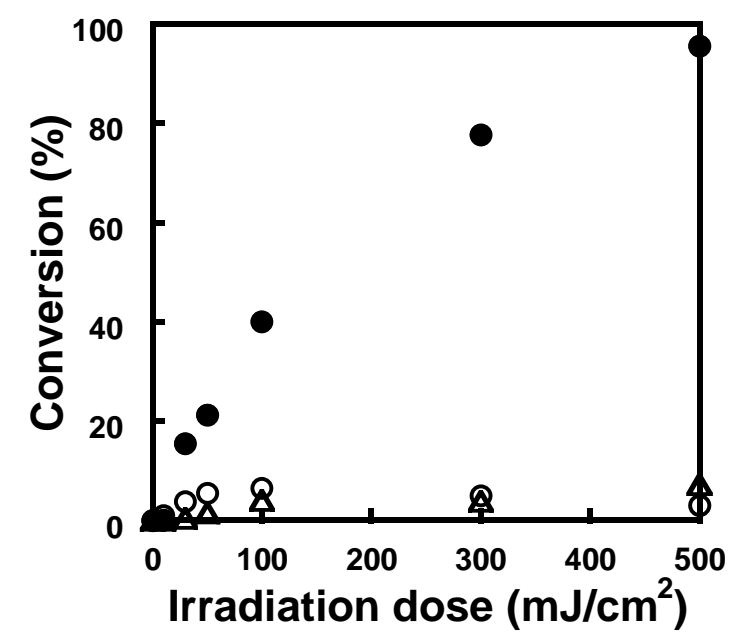

Fig. 5. Conversion of methacryl $(\mathrm{O})$ and chalcone $(\bullet)$ units of CDMA/EGDMA (1/1, wt/wt) blended film on irradiation at $365 \mathrm{~nm}$ in air. Conversion of methacryl unit $(\triangle)$ in EGDMA/TPO (1/0.03, wt/wt) blended film on irradiation at $365 \mathrm{~nm}$ in air was also shown.

The possibility of photocuring system with reduced oxygen inhibition using dimerization of the chalcone moiety was investigated. Sample mixture was coated on a $\mathrm{CaF}_{2}$ plate and the sample was irradiated in air. Figure 5 shows the conversion of the methacryl and chalcone units in CDMA/EGDMA (1/1, wt/wt) blended film on irradiation at $365 \mathrm{~nm}$ in air. Conversion of th methacryl unit of EGDMA/TPO (1/0.03, wt/wt) blended film on irradiation at $365 \mathrm{~nm}$ in air was also shown. The conversion of methacryl units of CDMA/EGDMA (1/1, wt/wt) was higher than that of EGDMA/TPO (1/0.03, wt/wt). Taking into account of the fact that photoradical polymerization of CDMA/EHMA (1/1, wt/wt) blended film proceeded on irradiation without photoradical initiators, the result showed the possibility of photocuring system with reduced oxygen inhibition using dimerization of the chalcone moiety. Improvement of the photopolymerization efficiency is now in progress.

\section{Acknowledgements}

This work was partly supported by the Grant-in-Aid for Scientific Research (C) No. 24550260 of the Japan Society for the Promotion of Science.

\section{References}

1. C. Decker, Polym. Int., 51 (2002) 1141.

2. J. V. Crivello, J. Polym. Sci. Part A: Polym. Chem., 45 (2007) 3759.

3. J. D. Oxman, D. W. Jacobs, M. C. Trom, V. Sipani, B. Ficek, A. B. Scrantony, J. Polym. Sci. Part A: Polym. Chem., 43 (2005) 1747.

4. M. Xiao, Y. He and J. Nie, Y. Des. Monomers Polym., 11 (2008) 383.

5. Y. He, M. Xiao, F. Wu and J. Nie, Polym. Int., 56 (2007) 1292.

6. Y. Cai and J. L. P. Jessop, Polymer, 47 (2006) 6560.

7. Y. Cai and J. L. P. Jessop, Polymer, 50 (2009) 5406.

8. R. Podsiad1y, K. Podemska and A. M. Szymczak, Dyes Pigments, 91 (2011) 422.

9. J.-D. Cho and J.-W. Hong, J. Appl. Polym. Sci., 93 (2004) 1473.

10. J. Zhou, Q. Zhang, H. Zhang, S. Chen and Q. Liu, J. Polym. Res., 19 (2012) 9984.

11. Y. Lin and J. W. Stansbury, Polymer, 44 (2003) 4781.

12. H. Wei, Q. Li, M. Ojelade, S. Madbouly, J. U. Otaigbe and C. E. Hoyle, Macromolecules, 40 (2007) 8788.

13. S. Li, Y. He and J. Nie, J. Photochem. Photobiol. A: Chem., 191 (2007) 25.

14. A. El-Ghayoury, C. Boukagtane, B. D. Ruiter and R. V. D. Linde, J. Polym. Sci. Part A: Polym. Chem., 41 (2003) 469.

15. K. Saigo, J. Synth. Org. Chem., Jpn., 47 (1989) 1006.

16. Y. Sonoda, Molecules, 16 (2011) 119.

17. M. Jayakannan, J. L. J. van Dongen and R. A.

J. Janssen, Macromolecules, 34 (2001) 5386.

18. A. Rehab, Eur. Polym. J., 34 (1998) 1845.

19. M. Tamilvanan, A. Pandurangan, B. S. Reddy and K. Subramanian, Polym. Int., 56 (2007) 104.

20. B. Y. Ban, S. Kaihua, J. H. Kim and D. H. Choi, Mol. Cryst. Liq. Cryst., 406 (2003) 93.

21. B. Waligora, M. Nowakowska and J. Kowal, Polym. J., 12 (1980) 767. 